\title{
Philosophiques
}

\section{Bolzano sur le temps et la persistance}

\section{Mark Textor}

Volume 30, numéro 1, printemps 2003

Bernard Bolzano. Philosophie de la logique et théorie de la connaissance

URI : https://id.erudit.org/iderudit/007734ar

DOI : https://doi.org/10.7202/007734ar

Aller au sommaire du numéro

\section{Éditeur(s)}

Société de philosophie du Québec

ISSN

0316-2923 (imprimé)

1492-1391 (numérique)

Découvrir la revue

Citer cet article

Textor, M. (2003). Bolzano sur le temps et la persistance. Philosophiques, 30(1), 105-125. https://doi.org/10.7202/007734ar

\section{Résumé de l'article}

Comment une proposition qui affirme que $a$ est fatigué le matin et n'est pas fatigué le midi peut-elle être vraie ? Bolzano soutient que toute proposition portant sur une chose contingente contient, dans la composante-sujet, la représentation d'un temps. Dans cet article, je reconstruis et évalue les arguments de Bolzano en les comparant à ceux de son adversaire principal, le tenant de la position selon laquelle toute proposition portant sur une chose contingente contient une copule renfermant la représentation du temps auquel l'objet représenté par la composante-sujet a la propriété représentée par la composante-prédicat (la conception de la modification de la copule). La conception bolzanienne de la modification du sujet ne peut résoudre le problème logique de la persistance qu'en assumant que des représentations-sujets qui contiennent différentes représentations de temps représentent différents individus dotés de déterminations temporelles. Mais ceci engendre une nouvelle question : comment un objet peut-il avoir différentes déterminations temporelles sans pour autant changer?
Ce document est protégé par la loi sur le droit d'auteur. L'utilisation des services d'Érudit (y compris la reproduction) est assujettie à sa politique d'utilisation que vous pouvez consulter en ligne.

https://apropos.erudit.org/fr/usagers/politique-dutilisation/ 


\title{
Bolzano sur le temps et la persistance ${ }^{1}$
}

\author{
MARK TEXTOR \\ King's College London \\ mark.textor@kcl.ac.uk
}

RÉSUMÉ. - Comment une proposition qui affirme que $a$ est fatigué le matin et n'est pas fatigué le midi peut-elle être vraie ? Bolzano soutient que toute proposition portant sur une chose contingente contient, dans la composante-sujet, la représentation d'un temps. Dans cet article, je reconstruis et évalue les arguments de Bolzano en les comparant à ceux de son adversaire principal, le tenant de la position selon laquelle toute proposition portant sur une chose contingente contient une copule renfermant la représentation du temps auquel l'objet représenté par la composante-sujet a la propriété représentée par la composante-prédicat (la conception de la modification de la copule). La conception bolzanienne de la modification du sujet ne peut résoudre le problème logique de la persistance qu'en assumant que des représentations-sujets qui contiennent différentes représentations de temps représentent différents individus dotés de déterminations temporelles. Mais ceci engendre une nouvelle question : comment un objet peut-il avoir différentes déterminations temporelles sans pour autant changer?

ABSTRACT. - How can we can truly say that $a$ is tired in the morning, and not tired at noon? Bolzano holds that every proposition about a contingent thing contains an idea representing a time in its subject-part. In this paper I reconstruct and assess Bolzano's arguments for his view of propositions about contingent things, comparing them to those of his main opponent, the view according to which every proposition about a contingent thing contains a copula combined with an idea that represents a time at which the object represented by the subject-part of the proposition has the property represented by the predicate-part (copula-modification view). Bolzano's subject-modification view could only solve the logical problem of persistence by assuming that subject-ideas containing different ideas of times represent different particulars bearing temporal determinations. But this creates a new question : how can an object have different temporal determinations and yet not change?

\section{Introduction}

La renommée de Bolzano auprès des philosophes analytiques est principalement redevable à ses multiples réalisations en philosophie de la logique. Sur la base de sa notion de proposition (en gros : la signification d'un énoncé assertorique), et d'une méthode qui consiste à faire varier les parties de la proposition, Bolzano (qui appelle ces parties «représentations») définit un concept de conséquence (sa déductibilité), ainsi qu'une notion de vérité

1. Cet article paraîtra en version originale dans History of Philosophy Quarterly et je tiens à remercier l'éditeure d'en avoir autorisé la publication en français. Je suis redevable pour plusieurs suggestions et critiques à : Ariana Betti, Philip Keller, Wolfgang Künne, Sandra Lapointe, Massimo Mugnai, Kevin Mulligan, Daniel Schulthess, Mark Siebel, Benjamin Schnieder, Armin Tatzel et un lecteur anonyme.

PHILOSOPHIQUES 30/1 — Printemps 2003, p. 105-125 
logique (son analyticité logique) qui s'apparentent manifestement aux définitions désormais célèbres de Tarski et de Quine ${ }^{2}$. Il est toutefois moins connu que Bolzano utilise aussi sa théorie des propositions pour définir une variété de notions épistémologiques et métaphysiques. Parmi les notions métaphysiques ainsi définies se trouve la notion de temps. La thèse cruciale en ce qui concerne la définition bolzanienne du temps est la suivante :

Un énoncé temporalisé ${ }^{3}$ du langage naturel qui attribue une propriété substantielle à une chose réelle exprime une proposition complète seulement s'il contient une expression telle que «à $t$ » comme partie de son terme-sujet.

Bolzano rejette par conséquent l'idée aristotélicienne que le temps se rattache aux prédicables ${ }^{4}$. La thèse de Bolzano suscite l'intérêt des philosophes contemporains parce qu'elle présente une ressemblance frappante avec les théories dans lesquelles des expressions telle que "Caius à midi » réfèrent à des parties temporelles. Cet article s'attache à la reconstruction et à l'évaluation de la partie de la doctrine des propositions de Bolzano qui est à la base de sa définition du temps. Cette définition elle-même constituera le thème de travaux ultérieurs, mais procédons par étapes : cette esquisse de la stratégie définitionnelle et du raisonnement de Bolzano introduira le lecteur au projet général de Bolzano en ce qui concerne le lien entre le temps d'un énoncé et le temps métaphysique.

C'est à la section $\mathbb{} 79$ de la Wissenschaftslehre, que Bolzano établit la connexion entre la thèse à propos du temps de l'énoncé et l'analyse du temps :

par le mot temps, nous ne pensons rien d'autre que cette détermination (Bestimmung) d'une chose réelle qui doit s'y trouver comme condition pour que nous puissions lui attribuer, conformément à la vérité, une certaine propriété (Beschaffenheit) ${ }^{5}$.

Les choses réelles sont celles qui entretiennent des relations causales, à savoir les substances et les adhérences (Adhärenzen) ${ }^{6}$. Les adhérences sont des propriétés individualisées (ce qu'on appelle aujourd'hui souvent les tropes) telles que la pâleur de Caius ${ }^{7}$.

2. Voir à ce sujet, respectivement, les articles de Mark Siebel et d'Edgar Morscher dans le même numréo. Note de l'éditeure.

3. Nous traduisons «tense» par «temps de l'énoncé», "tensed» et ses dérivatifs par " temporalisé » et ses dérivatifs.

4. Aristote défend cette conception dans De Interpretatione $16^{\mathrm{b}} 6$ et $16^{\mathrm{b}} 8$. Pour un plaidoyer plus récent, voir Geach, Peter T., Reference and Generality, Ithaca, Cornell University Press, 1962, \$2 27 et Wiggins, David, «Substance », dans Philosophy, dir. A. C. Grayling, Oxford, Oxford University Press, 1995, p. 232.

5. Bolzano, Bernard, Wissenschaftslehre, Sulzbach, Seidel, 1837, vol. 1, p. 365.

6. Sur le rôle des adhérences dans la sémantique bolzanienne, on consultera l'article de Benjamin Schnieder dans le même numéro. Note de l'éditeure.

7. En allemand, "wirklich» a la même racine que "wirken", (c'est-à-dire «causer»). En anglais, «actual» semble traduire « wirklich» de manière plus adéquate que le terme usuel, 
Dans la citation, la référence aux propositions est clandestine : lorsque Bolzano parle d'attribuer des adhérences aux choses existantes, il ne s'intéresse pas à une quelconque activité humaine, mais à la proposition exprimée par un énoncé de la forme « $\mathrm{A} a \mathrm{~b}$ » dans laquelle « $\mathrm{a}$ » est temporalisé. Selon Bolzano, un tel énoncé exprime une proposition de forme $[\mathrm{A} \text { à } \mathrm{t} a \mathrm{~b}]^{8}$. C'est là le point de départ de la compréhension bolzanienne de la notion d'un temps : ce à quoi l'adverbe de temps (ou, plus précisément, une de ses parties) réfère est un temps. Une représentation représentant un temps doit s'ajouter à chaque proposition du type sus-mentionné pour qu'on puisse l'évaluer comme vraie ou fausse.

La deuxième étape de Bolzano consiste à passer du niveau de la représentation au niveau des choses représentées. Il appelle un temps une "détermination " (Bestimmung) d'un objet réel. Il n'élucide nulle part la notion de détermination, mais, au sens minimum, une détermination est ce en vertu de quoi les choses réelles se distinguent les unes des autres. Les adverbes de temps et de lieu "attribuent» de telles déterminations. Les déterminations de temps sont, d'après Bolzano, similaires aux adhérences : tout comme ces dernières, les déterminations sont « liées » aux choses réelles. La formulation la plus claire de cet aspect de la théorie bolzanienne se trouve à la section $\$ 2.6$ de sa Versuch einer objektiven Begründung von den drei Dimensionen des Raumes : une détermination temporelle est rattachée à (haftet an) chaque objet réel.

Si on accepte ce que dit Bolzano jusqu'ici, on peut spécifier ce qui distingue les déterminations temporelles de tous les autres types de déterminations $^{9}$ : il n'y a en effet qu'une sorte de détermination qui doit adhérer à tout objet réel pour que cet objet soit le sujet d'une attribution vraie (fausse), à savoir la détermination qu'on trouve la réponse à une question de la forme "Quand A est-il b? ». Demander "Où Peter est-il intelligent? "n'a aucun sens. Mais la question : "Quand Peter est-il intelligent? " a un sens : L'est-il tout au long de sa vie? Au terme de ses études? etc. Le temps (ou l'éternité) se définit alors comme la collection de tous les temps ${ }^{10}$.

La stratégie définitionnelle de Bolzano est, de prime abord, attrayante : nous percevons le changement dans les objets, mais pas le temps auquel survient

\footnotetext{
à savoir «real ", parce qu'il évoque la causalité (= «to act»). Pour la traduction française, nous avons toutefois opté pour «réel »; non seulement la signification de «actuel » n'est-elle pas aussi opportune en français, mais la connotation temporelle du terme nous oblige, pour des raisons évidentes, à le rejeter et éviter ainsi de provoquer la confusion chez le lecteur. Note de la traductrice.

8. J'utilise les crochets pour former des noms de représentations et de propositions à partir des signes qui les expriment : $[\mathrm{A}]$ réfère à la représentation exprimée par « $\mathrm{A}$ ».

9. Versuch einer objektiven Begründung von den drei Dimensionen des Raumes, dans Bernard Bolzano, Gesamtausgabe, I/18 : Mathematisch-Physikalische und Philosophische Schriften, dir. Gottfried Gabriel, Matthias Gatzemeier et Friederich Kambartel, Stuttgart-Bad Cannstatt, Frohmann Holzboog, 1989 p. 228. (Citations d'après la pagination originale)

10. Bolzano, Versuch..., p. 229.
} 
ce changement. La définition de Bolzano ratifie cette intuition : les temps sont semblables aux entités théorétiques, pas aux entités observables ${ }^{11}$. Mais cette définition fait face à plusieurs problèmes : la transition du niveau des propositions et représentations au niveau des choses réelles est-elle justifiée? Avonsnous, en particulier, une conception indépendante et adéquatement justifiée de la détermination? Dans la négative, que gagnons-nous par cette transition? De plus, il n'est pas clair que la stratégie définitionnelle de Bolzano nous procure un concept de temps qui réussisse à faire tout ce à quoi Bolzano aspire : ce dernier soutient dans Versuch... que toutes les propriétés du temps peuvent être déduites de sa définition ${ }^{12}$. On peut en douter pour plusieurs raisons. George, par exemple, observe que l'ordre séquentiel du temps ne peut être déduit à partir de la définition de Bolzano ${ }^{13}$. Il vaut mieux prendre la définition bolzanienne du temps comme répondant à la question de savoir quelle sorte de choses sont un temps particulier et le temps en général ${ }^{14}$.

Après cette brève esquisse de la connexion entre la conception bolzanienne du temps de l'énoncé et du temps, revenons plus en détail sur la première. L'article procèdera comme suit : je clarifierai d'abord la thèse bolzanienne sur le temps de l'énoncé (section 1), pour ensuite la comparer avec quelques alternatives (section 2). La section 3 répondra à la question de savoir si la conception bolzanienne du temps de l'énoncé est plus adéquate que sa rivale aristotélicienne. Dans la section 4, je tenterai de reconstruire la conception bolzanienne de façon plus détaillée et je montrerai qu'elle rencontre plusieurs difficultés.

\section{Prédication et temps}

La théorie bolzanienne du temps part de l'observation qu'un énoncé assertorique attribuant une propriété b à une chose réelle A n'exprime une vérité complète que si elle spécifie quand $\mathrm{A}$ a $\mathrm{b}$.

Nous pouvons affirmer que chaque énoncé du type : L'objet réel A a (la propriété) $\mathrm{b}$ n'exprime une vérité complète que dans la mesure où nous relevons dans la représentation-sujet de cette dernière la détermination d'un temps donné. Ainsi, par exemple, les énoncés : "J'ai une sensation de douleur » ou «La terre est une planète» ne sont pas complètement vrais si on n'ajoute pas aux représentationssujets la détermination d'un temps donné, par exemple, «moi, à l'instant présent » ou «la terre, actuellement ». Et si on examine la chose de plus près, il devient évident, il me semble, que par le mot temps, nous ne pensons rien d'autre que cette détermination d'une chose réelle qui doit s'y trouver comme condition

11. Bolzano connaissait très bien la Critique de la raison pure de Kant. Kant utilise en B 48-49 une stratégie similaire pour définir le temps. Pour une discussion éclairante de la définition de Kant, voir von Wright, G.H., «Time, Change and Contradiction », dans Philosophical Papers II : Philosophical Logic, Oxford, Blackwell, 1983, p. 125s.

12. Bolzano, Versuch..., $\mathbb{S} 3$.

13. George, Rolf, «Bolzano on Time», Philosophia Naturalis, 24, 1987, p. 455s.

14. Bolzano explique une partie de son projet de cette façon dans Versuch... $\mathbb{} 2$. 
pour que nous puissions lui attribuer, conformément à la vérité, une certaine propriété $e^{15}$.

Dans cette citation, le propos de Bolzano semble vaciller entre les énoncés linguistiques et ce qu'ils expriment, c'est-à-dire les propositions (Sätze an sich). Je crois cependant qu'on peut, sans être téméraire, attribuer à Bolzano l'idée qu'un énoncé-type tel que (S1) :

(S1) La terre est une planète

n'est pas complètement vrai ou, pour reprendre la formule bolzanienne, n'exprime pas une vérité complète.

Mais, en quoi la thèse de Bolzano consiste-t-elle exactement ? Pour répondre à cette question nous devons examiner de plus près la conception bolzanienne de la vérité : à la section $\$ 125$ de la Wissenschaftslehre, il établit qu'être vrai est une propriété qui ne change pas en fonction des régions spatio-temporelles. Cette affirmation contraint Bolzano à endosser la position selon laquelle la vérité (fausseté) est une propriété stable des propositions. Le fait qu'il s'agisse d'une propriété stable ne règle toutefois pas la question de savoir s'il s'agit d'une propriété atemporelle ou éternelle, c'est-à-dire d'une propriété qu'une proposition a à chaque point du temps. Attaquons-nous maintenant à cette question.

Bolzano n'est pas un éternaliste à l'égard de la vérité. À la section $\$ 25$ de la Wissenschaftslehre, Bolzano expose ce qu'il croit être la compréhension correcte de l'idée de vérité éternelle : l'idée qu'une proposition est une vérité éternelle signifie que la proposition est une vérité et qu'elle attribue une propriété à quelque chose qui possède cette propriété en tout temps ${ }^{16}$. Cela peut toutefois encore nous induire en erreur : la possession d'une propriété mathématique devrait-elle, par exemple, être relative à des temps ? Le fait que Bolzano sente le besoin d'éliminer, à l'aide de paraphrases, les énoncés qui attribuent la vérité éternelle aux propositions montre cependant qu'il n'est pas un éternaliste à l'égard de la vérité. Sa paraphrase utilise seulement un prédicat de vérité monadique, l'adverbe de temps «éternel » qualifie l'instanciation de la propriété que la proposition attribue. De plus, lorsque Bolzano explique la signification de "vrai ", il ne classe jamais la vérité comme une relation, qu'elle soit éternelle ou non.

Bolzano est un atemporaliste aléthique : il soutient que la vérité est une propriété atemporelle des propositions. Chaque proposition, soit possède la propriété absolue attribuée par le prédicat monadique "est vrai", soit elle ne la possède pas; les propositions ne peuvent perdre, pas plus qu'elles ne peuvent acquérir cette propriété. Il n'est pas possible qu'une proposition vraie (fausse) perde cette propriété. Selon cette conception, Bolzano considère (S1) comme exprimant non pas une proposition, mais bien plutôt un fragment

15. Bolzano, Wissenschaftslehre, vol. 1, p. 365.

16. Ibid., vol. 1, p. 113. 
propositionnel. Car, la Terre peut être une planète et ne pas être une planète, à des temps différents (une catastrophe cosmique pourrait transformer la terre en une énorme météorite). Si elle doit exprimer une proposition, (S1) doit donc être complétée par quelque chose qui constitue une réponse à la question «Quand la terre est-elle une planète?».

Virtuellement personne n'admettrait qu'un énoncé-type tel que (S1) exprime une proposition complète. Mais, étant donné que le temps d'un verbe fonctionne comme un indexical temporel, il ne semble pas controversé de dire qu'une énonciation (utterance) de l'énoncé (S1) à un moment donné exprime quant à elle une proposition complète — sans l'ajout d'une expression de la forme «à $t$ ». Pourquoi Bolzano a-t-il donc besoin d'incorporer le sens d'une expression de la forme "à $t$ » au sein de la proposition exprimée par l'énonciation d'un tel énoncé?

La réponse de Bolzano est la suivante : les langues naturelles représentent le temps de façon trompeuse par le biais du temps de la copule : la copule «avoir» exprime le concept "pur» de la connexion entre un particulier et un attribut et, en plus de cela, elle représente un temps, et non, comme le suggèrent les langues naturelles, un concept de connexion relatif au temps ${ }^{17}$. La conception de Bolzano trouve d'ailleurs son support dans le langage ordinaire lui-même. Il arrive en effet que nous exprimions l'information temporelle que véhicule normalement la copule d'une manière différente; nous utilisons la copule et un adverbe de temps comme dans l'exemple : "Caius est ivre le matin ». La réponse à la question "Quand Caius est-il ivre? » se trouve dans la partie qu'on peut extraire de l'énoncé, à savoir "le matin », et non dans un certain caractère qu'on pourrait extraire du verbe. Bolzano considère que ces constructions correspondent à la structure de la pensée : la spécification temporelle est dissociée de la copule. La copule elle-même est alors comprise comme exprimant un concept non-temporalisé ${ }^{18}$. Bolzano considère que si la structure de la proposition exprimée doit être reflétée par l'énoncé qui l'exprime, tout énoncé de la langue naturelle dans laquelle une propriété substantielle est attribuée à une chose réelle doit être traduit dans un énoncé de la forme « $\mathrm{A}$ à $t \mathrm{a} \mathrm{b}$ » dans lequel la copule n'est pas temporalisée ${ }^{19}$.

\section{Les compléteurs temporels : ce qu'ils sont et ce à quoi ils servent}

Nous pouvons maintenant introduire la notion de compléteur temporel. Les exemples paradigmatiques de compléteurs temporels qu'utilise Bolzano sont des adverbes tel que "à $t$ ». Dans cette section, je m'intéresserai à la position de Bolzano sur la sémantique des compléteurs temporels, position selon laquelle ils modifieraient le sujet grammatical.

17. Ibid., vol. 2 , p. 5 .

18. La même stratégie pour éliminer le temps en utilisant les compléteurs temporels est employée par W. V. O. Quine dans Word and Object, Cambridge, Cambridge University Press, 1960, p. 172.

19. Bolzano, Wissenschaftslehre, vol. 2, p. 15. 
À quoi servent les compléteurs temporels? Par contraste avec les autres compléteurs, les compléteurs temporels devraient nous permettre d'attribuer des propriétés contradictoires à un même objet réel - bref, ils permettent de résoudre des contradictions. Selon Bolzano, les compléteurs temporels résolvent des contradictions de la manière suivante :

Les propositions qui ont des représentations-prédicats contradictoires ne peuvent être vraies que si elles ont des représentations-sujets distinctes. Ainsi, si deux attributs contradictoires (par exemple inculte et savant) doivent pouvoir être affirmés, conformément à la vérité, d'une seule et même substance (par exemple, Caius), les déterminations temporelles de la substance doivent être différentes, puisque si ces déterminations temporelles étaient les mêmes, deux propositions ayant la même représentation-sujet et des représentations-prédicats contradictoires devraient alors toutes deux être vraies ${ }^{20}$.

Que signifie tout cela? Nous comprenons des énoncés portant sur le changement, tels que (S2):

(S2) À midi, Caius était ivre, l'après-midi, Caius était sobre

comme non-contradictoires et nous les distinguons des énoncés, tel que (S3)

(S3) Caius était ivre et Caius était sobre.

qui, eux, le sont. Puisque (S2) et (S3) ne diffèrent qu'en ceci que (S2) contient des compléteurs temporels tel que "à midi ", la différence doit être imputée à la présence de compléteurs temporels. En revanche, les compléteurs spatiaux ne permettent pas de résoudre des contradictions. Lorsqu'on dit :

(S4) La voiture est brune devant et bleue derrière.

on parle de différentes parties de la voiture et non pas d'un objet avec des propriétés contradictoires.

Bolzano nous fournit encore davantage d'information en ce qui concerne les compléteurs temporels. Le sens d'un compléteur temporel (ou plutôt : la partie du sens qui correspond au «t») ne représente pas une adhérence. L'argument de Bolzano pour appuyer ce verdict se trouve à la section \$2.2 de Versuch..., et débute par une trivialité conceptuelle à propos du temps qui sera importante pour tout l'article : un objet peut persister de t 1 à t2 sans changer, c'est-à-dire sans perdre ni acquérir une adhérence monadique ou relationnelle. Si les compléteurs temporels représentaient des adhérences, cela ne serait pas possible.

Nous avons maintenant une idée de ce à quoi servent les compléteurs temporels. Mais comment réussissent-ils à remplir les tâches qu'on leur a assignées ? Le "problème formel de la persistance » consiste selon Johnstone à :

expliquer le rôle de la détermination temporelle dans nos attributions de changement, où expliquer ne signifie pas simplement opter pour une manière déterminée d'apposer des « $\mathrm{t}$ » et des « $\mathrm{t}$ * » mais à défendre des conceptions à propos 
des propriétés, de la nature du temps et de la nature des individus persistants qui justifient ce choix ${ }^{21}$.

Il y a quatre manières principales d'apposer « $t$ » et « $t$ * » à des énoncés. Elles résultent, lorsque combinées à la sémantique appropriée, dans différentes conceptions du temps et de la persistance :

1. Modification de l'énoncé : les compléteurs temporels doivent être représentés dans une version régimentée des langues naturelles par des opérateurs d'énoncés formant des énoncés. La forme régimentée d'un énoncé temporalisé est : "C'est le cas à t que : a est F ".

2. Modification de termes généraux : les compléteurs temporels génèrent, à partir de termes généraux n-adiques qui sont vrais de certains particuliers, des termes généraux $\mathrm{n}+1$-adiques. La forme régimentée d'un énoncé atomique temporalisé est : «tel que F-à-t ».

3. Modification de la copule : les compléteurs temporels sont des adverbes qui modifient la copule. La forme régimentée d'un énoncé atomique temporalisé est : "a est-à-t F ».

4. Modification du sujet: les compléteurs temporels modifient le sujet de l'énoncé. La forme régimentée d'un énoncé atomique temporalisé est : "a-à-t est $\mathrm{F}$ ».

La version contemporaine de la conception de la modification du sujet que présente Lewis va de pair avec une thèse métaphysique ${ }^{22}$. Un endurantiste soutient qu'une chose numériquement identique à elle-même peut exister à différents instants, alors qu'un perdurantiste conçoit cela comme impossible; seules les parties temporelles de ce que nous appelons à tort une "chose persistante " peuvent exister à des moments différents. La variante perdurantiste de 4 est :

4a. Modification du sujet perdurantiste : «à-t » est un opérateur de formation de termes qui prend le terme singulier correspondant à un particulier A et génère un terme singulier correspondant à la partie temporelle de $\mathrm{A}$ présente à $\mathrm{t}^{23}$.

Le problème logique de la persistance est d'expliquer comment les compléteurs temporels nous permettent d'attribuer des adhérences contradictoires à une même chose sans devoir accepter une contradiction. Un perdurantiste change les termes de la discussion. Il substitue à nos notions intuitives de chose persistante et de changement la notion de fusion des parties temporelles. La question de savoir si une conception justifiée des parties temporelles peut être développée est donc cruciale pour un perdurantiste, mais ce n'est pas une question à laquelle nous devons répondre ici.

21. M. Johnstone, "Is there a Problem about Persistence ? ", Proceedings of the Aristotelian Society, 61, 1987, p. 115.

22. Lewis, David, On the Plurality of Worlds, Oxford, Blackwell, 1986, p. 202.

23. Quine, Word and Object, p. 172. 
Bolzano soutient clairement, à la section $\$ 79$ de la Wissenschaftslebre de même que dans ses explications subséquentes sur le concept de temps, la conception de la modification du sujet. Selon Bolzano, le sens d'un compléteur temporel est contenu dans la représentation-sujet d'une proposition. Dans les langues naturelles, nous utilisons en effet des termes singuliers qui incorporent les compléteurs temporels. Des compléteurs temporels tels que «en 1946 » peuvent, soit modifier un verbe, soit faire partie d'une phrase nominale. La conception de la modification de la copule tient pour fondamentale la modification du verbe; Bolzano tient quant à lui pour fondamental le rôle de formateur de phrase nominale.

Bolzano est-il donc, à l'instar de Lewis, un perdurantiste? Difficile à dire. Les écrits de Bolzano sur le temps et le changement contiennent à la fois des éléments perdurantistes et endurantistes. À la section $\mathbb{} 45$ de la Wissenschaftslehre, il dit qu'un objet, à différents moments, est en fait un autre objet $^{24}$, ce qui ressemble à une acceptation prudente du perdurantisme. Mais Bolzano explique aussi fréquemment le changement comme étant un processus par lequel un seul et même sujet perd un attribut et en acquiert un autre ${ }^{25}$. Cette conception n'est compatible avec le perdurantisme que si on interprète ce qui est dit à propos du changement et du sujet persistant en termes perdurantistes. Il est toutefois frappant que Bolzano considère l'adjonction du sens d'un complément temporel à la représentation-sujet d'une proposition comme suffisante pour résoudre les contradictions du type concerné (voir la citation ci-dessus). Bolzano ne s'engage ici qu'à l'idée voulant que lorsque je dis : "Caius, le matin, était ivre; à midi, Caius était sobre ", deux représentations différentes de Caius ont été exprimées. Il laisse ouverte la question à savoir si les différentes représentations représentent des choses différentes. Si ajouter le sens d'un complément temporel à une représentation de Caius était suffisant pour résoudre le problème logique de la persistance, les théories de Bolzano au sujet du temps ne nous permettraient pas, à elles-seules, de le considérer comme un perdurantiste : nous pourrions toute de même prendre son affirmation qu'une substance est la même avant et après le changement au pied de la lettre. Si la résolution des contradictions du type concerné requiert plus qu'une différence au niveau des représentations-sujets, soit une différence dans ce qui est représenté, nous devons prendre les affirmations de Bolzano avec un grain de sel. On ne peut décider si Bolzano doit être considéré comme un endurantiste ou un perdurantiste qu'en examinant la plausibilité de ses positions sur la manière dont les contradictions sont résolues.

Mais avant de répondre à la question de savoir si Bolzano est un théoricien endurantiste du sujet, nous devons d'abord décider si les arguments de Bolzano en faveur de la conception de la modification du sujet sont convain-

24. Bolzano, Wissenschaftslehre, vol. 1, p. 202.

25. Bolzano, Bernard, Athanasia, oder Gründe für die Unsterblichkeit der Seele, Sulzbach 1827 , p. 82 ; et Wissenschaftslehre $\$ 91$. 
cants. Jusqu'à maintenant il semble plausible de dire qu'un énoncé qui exprime une proposition d'une façon logiquement transparente doit contenir un complément temporel. Mais nous n'avons pas vu d'arguments supportant l'affirmation que le sens d'un complément temporel doit faire partie de la représentationsujet. Étant donné les autres convictions philosophiques de Bolzano, l'alternative la plus plausible à sa conception est la troisième, à savoir, la conception de la modification de la copule. La première est incompatible avec la conception de Bolzano voulant que la vérité soit une propriété atemporelle de la proposition puisqu'elle requiert que la valeur de vérité de la proposition soit relative au temps. La seconde est incompatible avec l'idée intuitive souvent exploitée par Bolzano voulant que la substance perde et acquière des propriétés : une propriété comme être-vert-le-12-mars-1934 ne peut être perdue ou acquise. Voyons alors si principe de base de Bolzano sur la forme logique des propositions qui portent sur des choses réelles est plausible et, à cet effet, examinons ses arguments contre la conception de la modification de la copule.

\section{La conception de la modification de la copule}

À la section $\ 127.5$ de la Wissenschaftslehre, Bolzano pose la question suivante :

À travers la forme du verbe "avoir », ou de tout autre verbe qui inclut ce concept dans une proposition, le langage nous permet d'exprimer non seulement le genre et le nombre du sujet, mais aussi des déterminations temporelles [...]. Ne devrait-on pas en conclure que la véritable copule est formée non pas par le pur concept d'avoir, mais par un concept d'avoir combiné à la détermination d'un temps auquel quelque chose est eu ?26

Comme nous le verrons bientôt, Bolzano répond par la négative. À cet égard, Bolzano est indéniablement de son temps. La plupart des logiciens du dix-neuvième siècle considéraient que la copule exprime un concept non-temporalisé $^{27}$. Mais contrairement aux autres logiciens du dix-neuvième siècle, Bolzano place la complétion temporelle dans la représentation-sujet, et non dans la représentation-prédicat.

Cette idée d'une copule non-temporalisée était étrangère à Aristote. Ce dernier distinguait la copule des autres parties du discours précisément par le rôle qu'elle joue en co-signifiant le temps : du point de vue sémantique, les compléteurs temporels modifient la copule. Du point de vue ontologique, les objets ont des propriétés à des temps donnés, mais leurs propriétés ne sont pas liées au temps ${ }^{28}$. Les héritiers contemporains de l'approche aristotélicienne qualifient leur approche d' "adverbialiste ${ }^{29}$.

26. Bolzano, Wissenschaftslehre, vol. 2, p. 15.

27. Whateley, Elements of Logic, II, i, 2. Voir aussi, Prior, A. N., Time and Modality, Oxford, OUP/Clarendon, 1956; l'appendice A contient davantage d'informations historiques.

28. Cf. Haslanger, S., «Endurance and Temporary Intrinsics », Analysis, 49, 1989, p. 122.

29. Cf. Johnstone, M., "Is there a Problem about Persistence?"; Lowe, E.J., "The Problem of Intrinsic Change : Rejoinder to Lewis », Analysis, 48, 1988, p. 71-77; Haslanger, «Endurance and Temporary Intrinsics "; et Wiggins, «Substance». 
L'adverbialisme semble être la solution naturelle au problème logique de la persistance. Le temps est signifié, soit par une caractéristique de la copule, soit par un adverbe temporel qui modifie la copule. Par exemple, dans l'énoncé

Peter est présentement malade.

"présentement» modifie « est ». L'adverbe indique une manière dont la propriété peut être exemplifiée par un objet. Une personne qui est présentement malade n'a pas un type particulier de maladie : elle instancie ou exemplifie la maladie d'une manière particulière, à savoir présentement. "Présentement » appartient, d'après les adverbialistes, au même type d'adverbe que "nécessairement ». Quelqu'un qui est nécessairement un homme possède cet attribut d'une manière particulière ${ }^{30}$.

Selon certains adverbialistes, l'adverbe temporel «présentement » est le paradigme conceptuel du compléteur temporel. Dans un langage régimenté, un énoncé qui attribue une propriété substantielle à une chose réelle devrait avoir la forme canonique suivante :

$A$ est $t$-itement $b^{31}$.

Les adverbes de temps contribuent aussi à cette autre fonction que doivent remplir les déterminations temporelles : elles permettent de résoudre des contradictions et nous aident à décrire le changement d'une façon consistante. Tandis que :

(S5) Peter est malade et Peter est en santé

est une contradiction, l'énoncé :

(S6) Peter est présentement malade, mais était auparavant en santé

n'en est pas une.

Selon les adverbialistes, une énoncé $s$ contenant la copule et un compléteur temporel exprime une proposition vraie si et seulement si l'objet dénoté par $s$ instancie au moment ou pendant la durée introduit(e) par le compléteur la propriété dénotée par le nom de propriété. Selon ces spécifications des conditions de vérité, ni les porteurs de propriétés, ni les propriétés, ni les propositions ne sont relatifs au temps. C'est d'ailleurs ainsi qu'il doit en être.

L'adverbialisme nous permet d'expliquer une intuition sur laquelle Bolzano s'appuie à la section $\$ 2.2$ de Versuch... : les choses peuvent demeurer qualitativement les mêmes même si elles existent à des temps différents; l'écoulement du temps ne provoque pas, à lui seul, le changement. Les adverbialistes situent la relativisation du temps dans la copule : un même objet (numériquement parlant) peut exister à différents temps $\mathrm{t}$ et $\mathrm{t}$ * sans changer puisque le même objet peut exemplifier les mêmes propriétés aux temps $t$ et $t^{*}$.

30. D. Charles, Aristotle on Meaning and Essence, Oxford, Oxford University Press, 2000, Appendix 2, p. 384.

31. M. Johnstone, «The Problem of Intrinsic Change : Rejoinder to Lewis », p. 128. 
Seulement, il les instancie à t et $t$ *. Il n'y aurait pas non plus de problème à considérer le fait d'exemplifier une propriété à $t$ comme une propriété, puisqu'une chose ne peut perdre ou acquérir de telles propriétés.

L'adverbialisme peut de prime abord être séduisant, mais il fait néanmoins face à des difficultés insurmontables. Dans la Wissenschaftslehre, Bolzano avance, contre l'adverbialisme, les deux contre-arguments suivants :

(BA1) Je réponds que nous exprimons par le mot «avoir» une détermination temporelle, à savoir celle du temps présent, même lorsque nous parlons d'objets qui ne sont pas du tout dans le temps; par exemple, lorsque nous disons «chaque vérité - a - un objet sur lequel elle porte». Cela montre que nous ne sommes nullement justifiés de conclure de la connexion qu'établit le langage entre les déterminations temporelles et le concept d'avoir, à une relation essentielle entre eux.

(BA2)Si on doit indiquer clairement les parties d'un énoncé de la forme "L'objet A - a au temps $\mathrm{t}$ - la propriété $\mathrm{b}$ », il faut le faire de la façon suivante : «L'objet A au temps $\mathrm{t}-\mathrm{a}$ - (la propriété) $\mathrm{b}$ ». Car, ce n'est pas au temps $\mathrm{t}$ qu'on attribut la propriété $\mathrm{b}$ à l'objet $\mathrm{A}$, mais c'est à l'objet A en tant qu'il est conçu comme quelque chose qui se trouve au temps $\mathrm{t}$ (et donc pourvu de cette détermination) qu'on attribue la propriété $\mathrm{b}^{32}$.

Ces arguments sont-ils convaincants? (BA2) ne l'est pas, puisqu'il manifeste une mauvaise compréhension de l'adverbialisme. L'adverbialisme considère que les compléteurs temporels modifient la copule : l'instanciation ou l'exemplification est relative au temps. Selon Bolzano, l'adverbialisme affirme que les compléteurs temporels qualifient l'attribution de la propriété comme acte mental ou linguistique. Mais lorsque je dis, aujourd'hui :

(S7) Caius était ivre hier

l'acte iullocutoire d'attribution d'une propriété à Caius est exécuté aujourd'hui, mais la propriété est attribuée en tant qu'instanciée par Caius hier. L'instanciation de l'attribut est relativisée à un temps, mais pas l'attribution.

(BA1) s'adresse d'abord à l'idée voulant que la copule soit le locus de l'indication temporelle. Il vise à établir la thèse mentionnée à la section 2 comme quoi les langues naturelles représentent le temps de façon trompeuse. Les langues naturelles nous induisent en erreur dans certains cas : le temps présent de la copule introduit parfois une spécification temporelle là où il n'y a pas lieu d'y en avoir. Si je dis :

(S8) 7 est un nombre premier

je n'affirme pas, d'après Bolzano, que 7 est un nombre premier au temps présent ni que c'est un nombre premier à tous les temps. Le temps présent du verbe n'indique pas un temps, pas plus qu'il ne peut y avoir de compléteur temporel correspondant au temps présent dans une traduction logiquement

32. Bolzano, Wissenschaftslehre, vol. 2, p. 15. 
transparente de cet énoncé. Ce que j'affirme en énonçant (S8), c'est que 7 est un nombre premier, sans plus. Il y aurait par conséquent quelque chose comme exemplifier une propriété simpliciter.

Bolzano conclut de ces observations qu'il n'y a pas de lien essentiel entre la copule et le temps des énoncés. L'argument fonctionne aussi contre l'adverbialisme : l'adverbe temporel ne peut être considéré comme modifiant la copule. Il n'y a qu'une copule et elle exprime dans tous les énoncés le concept non-temporalisé d'avoir. Par conséquent, le fait que dans les langues naturelles les compléteurs temporels soient des adverbes ou des constructions adverbiales nous induit en erreur en ce qui a trait la structure logique. L'argument de Bolzano est que dans (S8), comme dans (S9)

(S9) Le bâton est courbé

il y a un élément commun : la copule pure. Bolzano cherche à maintenir la position selon laquelle il y a quelque chose comme avoir une propriété simpliciter. La conception de la modification du sujet est la seule qui, tout en étant consistante avec ses autres positions philosophiques, permette à Bolzano de le faire. Le compléteur temporel qui correspond au temps présent n'appartient pas à la copule, qui n'est pas temporalisée, mais au terme-sujet. Nous avons donc :

(S9-SV) Le bâton-à-cet-instant est courbé.

Le premier argument de Bolzano met au jour une réelle difficulté pour l'adverbialisme, puisque l'adverbialiste ne voudra pas admettre que les occurrences de «est» dans (S8) et (S9) expriment deux concepts d'instanciation qui n'ont aucun rapport entre eux. Demandons-nous alors en quoi consiste la différence entre ces concepts.

Le problème pour l'adverbialiste est que la copule exprimerait en (S8) un concept dyadique et en (S9) un concept triadique. Des adverbialistes tels que Lowe et Haslanger réagissent à ce problème en acceptant qu'il y ait un concept d'avoir simpliciter qui soit exprimé en (S8) et (S9). Leur idée est qu'avoir ou exemplifier est en fait une relation dyadique entre un objet et un attribut : avoir est une relation dyadique qui survient à un temps donné. Ce concept d'avoir simpliciter sert alors de concept primitif pour définir une relation triadique ${ }^{33}$. Le problème avec cette conception est qu'elle ne résout pas le problème de l'adverbialiste. Nous avons toujours deux concepts en $(\mathrm{S} 8)$ et $(\mathrm{S} 9)$ : un concept dyadique qui est nécessairement relatif au temps en (S9) et un concept dyadique simple en (S8). De plus, la décomposition de la version complétée de (S9) :

(S9') Le bâton est courbé à $\mathrm{t}$

33. Lowe, "The Problem of Intrinsic Change : Rejoinder to Lewis ", p. 74s; Haslanger, «Endurance and Temporary Intrinsics » p. 122; et Wiggins, «Substance », p. 232. 
ne peut pas être

(S9”) (i) Le bâton instancie la courbure (simpliciter) et

(ii) c'est le cas à t.

puisque, le cas échéant, les adverbes temporels ne pourraient plus résoudre les contradictions. La description d'un changement :

(S10) Le bâton est courbé à t et ce même bâton est droit à t*

reviendrait à :

(S10') (i) Le bâton instancie la courbure simpliciter et c'est le cas à t et

(ii) ce même bâton instancie la droiture simpliciter et c'est le cas à t*.

(S10') est contradictoire, puisqu'il implique que le bâton est courbé et droit simpliciter. Par conséquent, l'adverbialiste est forcé d'admettre que le fait d'avoir une propriété substantielle ne peut jamais être un avoir simpliciter où être analysé par le concours de cette notion ${ }^{34}$.

Cela montre aussi que nous ne pouvons nous débarrasser de la copule temporalisée en utilisant les compléteurs temporels. Dans les langues naturelles, les compléteurs temporels fournissent des informations supplémentaires, mais la copule, dans «Peter a la sagesse» ne devient pas non-temporalisée si on y ajoute "en $t$ ». Autrement, les compléteurs temporels ne pourraient résoudre les contradictions. Pour être plus précis : les compléteurs temporels ne peuvent résoudre les contradictions que si on les ajoute à des énoncés ayant une copule temporalisée.

La conclusion de cet argument crée un nouveau problème : nous sommes forcés d'admettre (contra Bolzano), soit (a) qu'il y a deux concepts d'avoir, soit (b) que le concept d'avoir simpliciter est un mythe philosophique $^{35}$. Même le concept d'avoir exprimé en (S8) entraîne une modification temporelle : 7 est en tout temps un nombre premier. Laquelle des alternatives devrait-on préférer?

Misons pour l'instant sur (a). Un partisan de (a) soutiendra que l'impossibilité de définir le concept atemporel sur la base du concept temporel n'implique pas que ces concepts soient sans aucun rapport. Leur relation n'est tout simplement pas celle qu'il y a entre un concept primitif et un concept défini. Je peux partir du concept d'avoir un attribut à un temps donné pour ensuite réaliser que certaines choses (tels que, par exemple, les nombres) ne peuvent changer. Il semble y avoir une tendance naturelle à dire alors que ces objets ont leurs propriétés à tous les temps. Cependant, une réflexion plus poussée sur les propriétés des nombres montre qu'elles ne sont pas simplement des propriétés éternelles, mais qu'elles sont essentiellement des propriétés éternelles. On pourrait alors se demander ce que «éternel» ajoute de plus à « essentiellement». Si un objet a une propriété essentiellement, il ne peut

34. Merricks, T., «Endurance and Indiscernibility ", Journal of Philosophy, 91, 1994, p. 169s. 35. Tichy, Pavel, "The Transience of Truth», Theoria, 46, 1980, p. 177s. 
perdre cet attribut; il devient donc superflu de dire qu'il a cet attribut à tous les temps. On adopte ainsi une copule atemporelle d'avoir un attribut essentiellement. Cette copule ne peut être définie dans les mêmes termes qu'une copule temporelle; mais nous avons donné un scénario de prime abord plausible de la manière de parvenir à ce concept par une réflexion sur le concept temporellement relativisé. Il s'ensuit que l'adverbialiste n'introduit pas des concepts d'avoir qui n'ont aucune rapport entre eux et qu'il peut tout de même expliquer la relation intime qu'ils entretiennent. En bref: Bolzano n'a pas d'argument convainquant contre l'adverbialisme. Mais sa solution de rechange, telle que nous l'avons esquissée plus haut, est peut-être néanmoins plus intéressante. Voyons cela.

\section{La conception de la modification du sujet}

Voici encore une fois les principes de base de la position de Bolzano :

(B1) Chaque énoncé de la langue naturelle attribuant une propriété à une chose réelle qui exprime une proposition d'une façon qui reflète la structure de cette dernière contient un compléteur temporel de la forme «à $t$ ».

(B2) Le sens d'un compléteur temporel fait partie de la représentation-sujet de la proposition exprimée par un tel énoncé.

(B3) «A-à-t » et "A-à-t* » expriment différentes représentations-sujets.

Le problème est à savoir si (B1)-(B3) sont suffisants pour générer une réponse au problème logique de la persistance ou si on doit bien plutôt y ajouter l'hypothèse que « $\mathrm{A}$-à- $\mathrm{t}$ » et «A-à- $\mathrm{t}$ * " expriment des représentationssujets différentes représentant des objets différents.

Voyons d'abord sur quels fondements repose la position de Bolzano. Puisqu'il y a, comme nous l'avons vu, des options intéressantes, nous sommes en droit d'exiger de tels fondements. À la section $\$ 183$ de la Wissenschaftslehre, Bolzano fait appel au rôle que remplissent les compléteurs temporels lorsqu'il s'agit de résoudre des contradictions pour appuyer sa position ${ }^{36}$ :

(P1) Tout énoncé qui exprime de façon logiquement transparente une proposition qui attribut une propriété substantielle à une chose réelle contient un compléteur temporel.

(P2) Un compléteur temporel peut résoudre des contradictions seulement si son sens fait partie de la représentation-sujet de la proposition.

(P3) Les compléteurs temporels permettent de résoudre des contradictions.

(C) Le sens d'un compléteur temporel fait partie de la représentation-sujet d'une proposition.

La prémisse cruciale de cet argument est (P2). Mais, pourquoi devrions-nous l'accepter? Une réponse partielle est que le compléteur temporel ne peut être 
conçu comme attribuant une propriété (ou quelque chose de semblable) à un particulier, c'est-à-dire qu'il ne peut être un prédicat ou quelque chose d'analogue. Ainsi, le sens d'un compléteur temporel doit être conçu comme partie de la représentation-sujet qui, elle, n'attribut pas de propriété. La prémisse de cet argument est vraie : supposons, pour les fins d'une réduction, que «A-à$\mathrm{t}$ » signifie la même chose que «A, qui existe à $\mathrm{t}$ ». Récemment, Merricks a plaidé pour la même idée de la façon suivante :

« $\mathrm{o}$ à $\mathrm{t}$ » réfère à l'objet qui est o et qui existe à $\mathrm{t}$; il ajoute à tout énoncé qui le contient le fait que o existe à $\mathrm{t}^{37}$.

Bolzano s'interroge sur cette hypothèse à la section $\mathbb{\$} 183$ de la Wissenschaftslehre. Dans la cette section, il tente de montrer que les représentations exprimées par «A à $t$ » et «A qui existe à $t$ » ne sont pas même coextensives. Son argument procède comme suit :

(P1) Si x est une substance (simple), $\mathrm{x}$ existe à tous les temps, si x existe à un moment.

(K1) Si la dénotation de "A qui existe à $t$ » est une substance, la partie «qui existe à $t$ » est redondante dans «A qui existe à $t$ ».

(P2) $\mathrm{Si}$ « A qui existe à $\mathrm{t}$ » et « $\mathrm{A}$ à $\mathrm{t}$ » sont co-extensives, «à $\mathrm{t}$ » devrait être redondant dans «A à $t$ "

(P3) «à t » dans «A à t» n'est pas redondant

$(\mathrm{K} 2)$ «A qui existe à $\mathrm{t}$ » et « $\mathrm{A}$ à $\mathrm{t}$ » ne sont pas co-extensives

La notion de redondance employée ici est d'ordre technique : une représentation $i$ est une partie redondante d'une représentation $j$ si et seulement s'il y a une représentation $j^{*}$ qui contient, dans le même ordre, les mêmes représentations que $\mathrm{j}$ à l'exception de $\mathrm{i}$, et $\mathrm{j}^{*}$ représente le même objet que $\mathrm{j}^{38}$. En gros, une représentation est une partie redondante si et seulement si on peut la laisser tomber sans que cela n'affecte la référence de la représentation dont elle fait partie. L'argument de Bolzano présente deux problèmes :

Premièrement, Bolzano considère que $(\mathrm{P} 1)$ découle du concept de changement $^{39}$. Mais cet argument est fallacieux. Même si nous concevons le changement comme quelque chose qui affecte un objet qui existe avant et après le changement, il n'y a pas lieu de penser qu'il y a une chose qui existe avant et après tout changement. La prémisse qui dit que les substances sont éternelles semble plus controversée que l'hypothèse dont elle devrait permettre de démontrer la véritét0.

37. Merricks, «Endurance and Indiscernibility», p. 183.

38. Bolzano, Wissenschaftslehre, vol. 1, p. 309s.

39. Bolzano, Athanasia, p. 82.

40. Pour une discussion plus approfondie de l'argument bolzanien en faveur de l'éternité des substances, voir Textor, Mark, «Bolzano über die Unvergänglichkeit der Seele », dans Bolzanos Erbe für das 21. Jahrhundert, dir. E. Morscher, St. Augustin, Academia, 1999, p. 269-294. 
Deuxièmement, pourquoi devrions-nous accepter (P3) ? Si «A à t " réfère à $\mathrm{A}$ et non à la partie temporelle de $\mathrm{A}$, «à $\mathrm{t}$ " est redondant. On le remplace sans changer l'extension de l'expression. Bolzano semble donc se voir forcé de refuser (P3).

Heureusement, il y a un argument plus direct contre l'idée qu'on doit interpréter «à $\mathrm{t}$ » comme voulant dire «existe à $\mathrm{t}$ ". L'interprétation de «à $\mathrm{t}$ » comme un prédicat d'existence temporalisé n'élucide pas le problème logique de la persistance : l'ajout de tels prédicats d'existence ne résout pas les contradictions qui nous intéressent ici, puisque :

Caius qui existe à $t$ est ivre et Caius qui existe à t* n'est pas ivre.

est contradictoire. Cet énoncé dit que Caius existe à t et t* et qu'il est ivre et non-ivre. De plus, "qui existe à $t$ » ne peut servir à compléter l'expression d'une proposition si l'énoncé en question est déjà grammaticalement complet. L'expression :

Caius qui existe maintenant est ivre

est l'expression incomplète d'une proposition. Sans détermination temporelle, sa valeur de vérité peut changer : Caius qui existe maintenant peu être ivre et sobre à différents instants. Notre première tentative endurantiste pour comprendre " $\mathrm{A}$ à $\mathrm{t}$ » échoue donc. Cela bien sûr ne constitue pas une preuve de la vérité de la conception de la modification du sujet de Bolzano. Même si le compléteur temporel «à $\mathrm{t}$ » n'est pas un prédicat d'existence temporalisé, il peut tout de même être ce à quoi il ressemble : un adverbe modifiant la copule. Si c'est bel et bien là son rôle, il peut à la fois être un compléteur temporel et permettre de résoudre des contradictions. Ainsi, Bolzano n'a pas d'argument convaincant en faveur de la supériorité de sa position.

Mais peut-être la théorie de Bolzano est-elle meilleure que la théorie adverbiale. Malheureusement, si «à $t$ » n'est pas interprété comme un opérateur formant des termes qui désignent des parties temporelles, rien n'est moins clair que la manière dont il modifie le sujet grammatical de l'énoncé. Une analogie avec l'attribution du prédicat «bon» permet de clarifier l'idée que Bolzano cherche à cerner en faisant de «à $t$ » une partie du sujet grammatical. Le premier point de l'analogie avec l'attribution du prédicat «bon » est :

1. Nous ne pouvons dire que A est bon ou mauvais sans informations sortales ou fonctionnelles additionnelles à propos de $\mathrm{A}$.

2. Nous ne pouvons dire que A est vert ou non-vert, sans informations temporelles supplémentaires à propos de $\mathrm{A}$.

La raison pour laquelle 1 . et 2 . sont valables sont là encore analogues :

3. Il est possible que A soit bon et mauvais.

4. Il est possible que A soit vert et non-vert.

Dans le cas du prédicat «bon ", l'apparente contradiction est résolue en spécifiant l'espèce à laquelle appartient la chose en question. Par exemple, A 
est un bon menuisier et un mauvais cuisinier. Nous pouvons attribuer le prédicat "bon" (ou son contraire) à un objet seulement si nous nous savons à quel type d'objet il appartient. Il n'y a rien de tel que «être bon» simpliciter.

La contradiction est résolue si nous rendons explicite ce qui est dit ou communiqué de façon pragmatique par «A est bon» et que nous l'élargissons pour obtenir, par exemple, "A est un bon menuisier ", car l'énoncé ainsi élargi ne peut être fragmenté pour donner la conjonction «A est bon et est menuisier ». Geach a forgé pour les adjectifs qui mettent en évidence cette caractéristique le terme «adjectif attributif » ${ }^{41}$. Dans «A est un bon menuisier », il n'y a pas de prédicat conjonctif. Dans le langage ordinaire, nous avons une autre façon de diviser la combinaison d'un adjectif attributif et d'un prédicat sortal :

A comme menuisier est bon, mais comme cuisinier mauvais.

L'apposition "comme menuisier» fournit des informations sortales ou fonctionnelles à propos de $\mathrm{A}$, mais elle ne change pas la référence du sujet grammatical. Pouvons-nous comprendre «A-à- $t$ » par analogie avec «A comme menuisier ${ }^{42}$ ?

Cela n'est guère évident. Si A comme menuisier est bon, c'est qu'il est menuisier (en termes bolzaniens : il a l'adhérence de menuisier-ité) ${ }^{43}$. Les appositions au sujet grammatical spécifient des adhérences, mais «à $t$ » ne peut spécifier une adhérence de A. Cela découle clairement de la section 2. Autrement, aucun sujet ne pourrait durer et demeurer qualitativement le même. Ainsi, l'analogie ne fonctionne pas et ne nous aide pas à comprendre la sémantique bolzanienne pour « $a-a ̀-t$ ».

Nous n'avons toujours pas d'explication sémantique des termes singuliers tels que «a-à-t » qui soit compatible avec l'endurantisme. Mais nous pouvons, même sans une telle explication, tout de même voir que l'idée de Bolzano, à savoir que le sens d'un compléteur temporel fait partie de la représentationsujet de la proposition fait face à un sérieux problème en ce qui concerne le rôle que jouent les compléteurs temporels dans la résolution des contradictions. À la section $\$ 79$ de la Wissenschaftslehre, Bolzano nous disait que deux propositions qui attribuent des propriétés contradictoires à un même objet ne sont pas contradictoires si leurs représentations-sujets sont différentes. Mais comment la différence dans les représentations-sujets de deux propositions peut-elle résoudre une contradiction si elle n'entraîne pas que les deux propositions portent sur des objets différents? Il est vrai que lorsque $a$ et $b$ sont un même objet, une conjonction telle que [a est $\mathrm{F}$ et $\mathrm{b}$ n'est pas $\mathrm{F}$ ], peut ne pas être manifeste-

41. Geach, Peter T., "Good and Evil», dans Theories of Ethics, dir. Philippa Foot, Oxford, Oxford University Press, 1967, p. 64.

42. Pour une tentative plus récente, voir D. L. M. Baxter, "Identity through Time and the Discernibility of Identicals ", Analysis, 49, 1989.

43. Sur l'utilisation de la forme schématique «F-ité » pour désigner les adhérences, on peut consulter la section 3 de l'article de Benjamin Schnieder dans le présent numéro. Note de l'éditeure. 
ment contradictoire. Prenons un exemple : même si Hespérus est la même planète que Phosphorus, la proposition conjonctive [Hespérus est une planète et Phosphorus n'est pas une planète] n'a pas la forme logique [p et non-p]. Bien que la conjonction soit nécessairement fausse, on peut y croire sans être totalement irrationnel. [Caius-le-matin est ivre et Caius-à-midi n'est pas ivre] est alors aussi une proposition conjonctive qui peut être le contenu d'une croyance rationnelle. Mais selon le concept intuitif de changement, cette proposition est vraie et non pas nécessairement fausse! Comment cela peut-il être le cas si Caius-le-matin est la même personne que Caius-à-midi et que seules les représentations-sujets [Caius-le-matin] et [Caius-à-midi] sont différentes? Comment peut-on soutenir que Caius-le-matin est ivre simpliciter, alors que Caius-à-midi ne l'est pas?

Il semble alors ne pas y avoir de compréhension de «a-à- $t$ » qui permette de résoudre le problème logique de la persistance et qui soit compatible avec l'endurantisme. Si nous désirons fournir une reconstruction opportune de la théorie de Bolzano, nous devons donc renforcer les éléments perdurantistes de sa position. Nous devons considérer sérieusement l'assertion de Bolzano qui dit que les objets à des temps différents sont des objets différents. [Caius-à-t] et [Caius-à-t*] représentent différents objets, mais lesquels ?

Afin de répondre à cette question, nous pouvons examiner les passages perdurantistes dans l'œuvre de Bolzano. Dans un énoncé de la forme «A à t est $\mathrm{b}$ »,

c'est à l'objet A en tant qu'il est conçu comme quelque chose qui se trouve au temps $t$ (et donc pourvu de cette détermination) que nous attribuons la propriété $b^{44}$.

Ce qui est dit lorsqu'on énonce " $\mathrm{A} \mathrm{a} \mathrm{b}$ " à $\mathrm{t}$ est que $A$ ayant $t$ est $\mathrm{b}$. Bolzano traite $A$ ayant $t$ et $A$ ayant $t$ * comme des objets distincts. Ils sont constitués en appliquant des déterminations de temps différentes au même objet réel. On peut présumer que c'est là la raison Bolzano dit, à la section $\mathbb{S}$ 45 de la Wissenschaftslehre, que « un objet, à un autre temps différent, est en fait un autre objet ».

Cela nous aide-t-il à solutionner le problème logique de la persistance? Non. Cela ne fait que changer le mal de place. Souvenons-nous qu'à la section $\mathbb{} 2.2$ de Versuch..., Bolzano s'en remet au sens commun : une chose réelle peut exister à différents moments sans changer. La conception bolzanienne du temps comme attribut spécial des objets réels est incompatible avec cette intuition. Nous devons alors nous demander pourquoi différentes déterminations temporelles peuvent se rattacher à un même objet sans que cela ne constitue un changement. L'analogie avec les adhérences, dans ce cas, ne tient plus. Si on met l'accent sur les éléments perdurantistes de l'approche bolzanienne, on résout un problème en en créant un autre : qu'ont donc de particulier les déterminations 
et la relation qui les fixe aux objets pour qu'on puisse y fixer différentes déterminations temporelles sans que cela n'entraîne un changement?

Contrairement à la conception de la modification du sujet, l'approche adverbiale semble bien équipée pour résoudre le problème logique de la persistance :

1. Nous avons une compréhension intuitive des modifications adverbiales dans les langues naturelles. Nous utilisons et comprenons les adverbes des langues naturelles tels que «maintenant» et «le jeudi».

2. L'approche adverbiale est métaphysiquement satisfaisante : les déterminations de temps ne sont pas, selon l'adverbialisme, de mystérieux attributs spéciaux des particuliers, mais des façons d'avoir une propriété. Cela est davantage conforme à la conception intuitive du changement et de la persistance.

Ainsi, l'adverbialisme s'accorde mieux aux intuitions de Bolzano à l'égard du temps que sa propre théorie. Comment l'adverbialiste devrait-il alors traiter les termes singuliers tels que «Paris au printemps» qui ont inspiré la suggestion de Bolzano?

Il doit les concevoir comme des dérivés des adverbes qui modifient l'instanciation d'une propriété. Prenons un exemple:

Caius adolescent est souvent ivre, Caius dans la vingtaine ne l'est pas

On ne peut comprendre cet énoncé que si on peut répondre à des questions telles que "Quand Caius est-il souvent ivre? » La réponse est : "Caius est souvent ivre à l'adolescence et Caius n'est pas souvent ivre dans la vingtaine». Dans une réponse de cette espèce, les adverbes de temps se substituent à ces curieux termes singuliers qu'implique la conception bolzanienne. L'explication doit selon moi être comprise comme allant des adverbes de temps vers les termes singuliers et non l'inverse. Quiconque ne maîtrise pas les adverbes de temps ne peut comprendre les propos de Bolzano.

Voici un autre exemple qui illustre cette idée. Si «a-à-t » (et ainsi de suite) sont des termes-sujets complexes, des énoncés comme celui qui suit sont bien formés et ont une signification :

La Vienne du siècle dernier est identique à la Vienne de ce siècle.

Mais la seule façon de comprendre cela littéralement est de la comprendre comme l'affirmation que Vienne existe maintenant et existait déjà au siècle dernier. Là encore, les compléteurs temporels fonctionnent comme adverbes, et cela est juste et bon.

\section{Conclusion}

Bolzano fait de brillants efforts pour montrer que toutes les propositions incorporent le même concept pur reliant deux représentations : les énoncés temporalisés expriment des propositions ayant des représentations-sujets spéciales, et la copule est la même que dans les énoncés non-temporalisés. Nous 
avons tenté de reconstruire les arguments de Bolzano en faveur de cette position et en avons montré les insuffisances. Bolzano devrait être vu comme adoptant une position voisine du perdurantisme. Le rôle des parties temporelles est rempli, dans son ontologie, par un objet réel qui porte une détermination de temps. Le problème avec cette idée est que notre notion de changement et de persistance semble être plus fondamentale et plus claire que la notion bolzanienne d'une détermination temporelle fixée à un objet réel. Ces résultats négatifs affaiblissent la définition bolzanienne du temps. Quant à savoir si la stratégie de Bolzano peut néanmoins être employée avec succès, elle devra, comme nous l'avons dit précédemment, faire l'objet d'un autre article.

(Traduit de l'anglais par Mélissa Thériault et Sandra Lapointe) 